\title{
CRITÉRIOS DE DIAGNÓSTICO DE ESCLEROSE MÚLTIPLA
}

\author{
ANTÓNIO MAGALHÃES, VASCO SALGADO \\ Laboratório de Bioquímica e de Imunologia do Centro de Estudos Egas Moniz. Serviço de Neurologia do Hospital de Santa Maria. Lisboa
}

\begin{abstract}
RESUMO
Os autores apreciam o problema do estabelecimento de critérios de diagnóstico de E. M., que se mostrem adaptados às actuais investigações sobre a doença e aos recentes progressos laboratoriais considerados úteis auxiliares clínicos. Salientam a importância da classificação dos casos estudados em grupos com diversos graus de probabilidade diagnóstica. A integração nos critérios das alterações do L. C. R., em particular dos resultados da análise imunitária, é posta em relevo. Pensam, porém, que o valor destes dados, entre os restantes critérios, deve ser avaliado segundo justa medida. Assim, no momento actual, não julgam possível considerá-los obrigatórios para o diagnóstico definitivo de $\mathrm{E}$. M. Finalmente, apresentam o esquema de critérios que utilizam.
\end{abstract}

\section{SUMMARY}

\section{Diagnostic criteria of multiple sclerosis}

The authors discuss diagnostic criteria of multiple sclerosis fitted to the present research and the recent advances in clinical laboratory methods. The importance of classifying the cases to be studied in groups of grading probability of diagnosis is emphasized. No less decisive is the integration of laboratory findings into the diagnostic criteria, especially those resulting from the modern examination of cerebrospinal fluid immunitary analysis. But it must be pointed out that the great clinical significance of these aspects does not imply to consider them obligatory for definitive diagnosis of multiple sclerosis. The criteria used by the authors are indicated. One may say they integrate principles and criteria successively suggested by Schumacher, Rose, and Bauer. Some main points are referred: 1. Cases classification as in Bauer's criteria: a) multiple sclerosis proved by autopsy; b) clinically definite multiple sclerosis; c) probable multiple sclerosis; d) possible multiple sclerosis. 2 . The characteristic cerebrospinal fluid profile is not considered obligatory but frequent in clinically definite multiple sclerosis group. 3 . The three last groups are divided in two subgroups: recent cases (apparent duration of the illness no longer than one year) and no recent cases.

A Esclerose Múltipla (E. M.), também chamada Esclerose em Placas, é uma grave doença do sistema nervoso, de etiopatogenia e terapêutica específica desconhecidas, que constitui objecto de intensiva investigação multidisciplinar, no momento presente.

Obviamente, os diversos aspectos de tal estudo exigem rigor diagnóstico; e mais: uma universal concordância dos critérios de diagnóstico. Ora reconhece-se que em elevado número de casos - nomeadamente, casos recentes, ou no decurso das primeiras observações clínicas - não é possível efectuar um diagnóstico seguro e definitivo, mas tão somente diagnóstico de probabilidade.

Em especial, durante as duas últimas décadas, impôs-se a necessidade de rever e de aperfeiçoar os critérios de diagnóstico da E. M.

Uma Comissão presidida por Schumacher elaborou um conjunto de critérios clínicos aos quais deve obedecer o diagnóstico definitivo de E. M. ${ }^{1}$ Kurtzke $^{2}$ sintetizou-os do modo seguinte:

1. O exame neurológico deve revelar alterações objectivas que possam ser atribuidas a disfunção do sistema nervoso central.

2. O exame objectivo ou a história clínica devem indicar lesão de duas ou mais áreas do sistema nervoso central.
3. A evidência de doença do sistema nervoso central deve apontar para lesões predominantes na substância branca.

4. A evolução das lesões no sistema nervoso central deve seguir um dos dois padrões seguintes:

a) Dois ou mais episódios de agravamento, cada um com duração igual ou superior a vinte e quatro horas e com intervalos não inferiores a um mês.

b) Progressão lenta ou gradativa dos sintomas neurológicos durante um período mínimo de seis meses.

5. As primeiras manifestações da doença devem ocorrer entre os dez e os cinquenta anos de idade.

6. Ausência de outro diagnóstico neurológico mais provável.

Os critérios da Comissão Schumacher foram considerados de grande utilidade, quando se pretendia seleccionar doentes com diagnóstico definitivo, como sucede na avaliação de meios terapêuticos. Contudo, o mesmo esquema mostrou-se notoriamente insuficiente em estudos de diferente natureza, tais como investigações que visam esclarecer aspectos etiopatogénicos. Neste domínio, a aplicação daqueles critérios conduz a excluir casos susceptíveis de fornecerem dados relevantes, designadamente, os casos mais recentes eventualmente, os primeiros surtos - aqueles em que com 
maior frequência o diagnóstico é menos preciso. Se é certo que os resultados de vários estudos indicam, pelo menos em parte dos doentes, a ocorrência de fases evolutivas com distintas características biológicas e diferenças na importância dos factores etiopatogénicos implicados, compreende-se a utilidade de poder dispor de tais casos.

Esta visão dos problemas determinou que o diagnóstico de $\mathrm{E}$. M. fosse encarado numa perspectiva dinâmica: os doentes seriam classificados segundo o grau de probabilidade diagnóstica e submetidos a observações seriadas, periódicas, ou sempre que surgisse agravamento. Por meio de sucessivos exames, o diagnóstico pode ganhar maior consistência ou perdê-la: ao mesmo doente podem ser atribuídos diferentes graus de probabilidade, entre o diagnóstico definitivo e a sua exclusão.

É concretizado este conceito no esquema proposto por Rose et al. $^{3}$ Os autores consideraram três graus de probabilidade diagnóstica da E. M., definidos por critérios clínicos, como seguidamente se apresenta.

\section{E. M. com diagnóstico clínico definitivo:}

1. Evolução recorrente e remissiva, pelo menos com dois surtos, separados por um intervalo não inferior a um mês; ou

2. Evolução lenta ou gradativamente progressiva, durante, pelo menos, seis meses.

3. Sintomas objectivos verificados, atribuíveis a lesão de mais de uma área do sistema nervoso central, predominantemente da substância branca.

4. Habitualmente, início dos sintomas entre as idades de dez e cinquenta anos.

5. Ausência de melhor explicação neurológica.

II. E. M. provável:

1. História de sintomas recorrentes e remissivos, mas sem verificação objectiva, com manifestações neurológicas frequentemente associadas à E. M.; ou

2. Observação de sintomas de um único surto, constituídos por sinais de lesão multifocal da substância branca do sistema nervoso central, com boa recuperação, eventualmente seguidos de outra sintomatologia.

3. Ausência de melhor explicação neurológica.

III. E. M. possivel:

1. História de sintomas recorrentes e remissivos, sem verificação clínica; ou

2. Observação de sintomas insuficientes para evidenciarem mais de uma lesão na substância branca do sistema nervoso central.

3. Ausência de melhor explicação neurológica.

Como se observou, os critérios de Rose afastam os limites etários entre os quais podem surgir as primeiras manifestações da doença (dez e cinquenta anos no esquema de Schumacher), permitindo a inclusão de casos de E. M. infantil e de doentes com início aparente tardio.

$O$ desenvolvimento de métodos laboratoriais que se mostraram úteis auxiliares de diagnóstico exigiu uma nova revisão dos critérios. Salientam-se, além das análises tradicionais do líquido céfalo-raquidiano (L. C. R.), exames imunoquímicos deste líquido que permitem evidenciar ou sugerir uma reacção imunitária no sistema nervoso central.

Em comunicação a um simpósio internacional (Progress in MS - Research), que teve lugar em Göttingen, no âmbito das actividades da Internacional Federation of Multi- ple Sclerosis Societies, Bauer ${ }^{4}$ propôs um conjunto de critérios de diagnóstico, no qual, apoiando-se nos conceitos de Rose, inclui os dados fornecidos pelos exames do líquor, conferindo-lhes especial relevo. Segue-se o referido esquema.

I. E. M. comprovada por autópsia.

II. E. M. com diagnóstico clinicamente definitivo:

1. Evolução recorrente e remissiva com, pelo menos, dois surtos separados por intervalo não inferior a um mês; ou

2. Evolução lenta ou gradativamente progressiva durante, pelo menos, seis meses.

3. Sintomas neurológicos verificados, atribuíveis a lesão de mais de uma área do sistema nervoso central, predominantemente da substância branca.

4. Alterações características do L. C. R.:

Pleocitose mononuclear; aumentos das concentrações de globulina gama e de imunoglobulina $\mathrm{G}$, com distribuição oligoclonal e indicações de síntese no sistema nervoso central.

5. Habitualmente, início dos sintomas entre os dez e os cinquenta anos de idade.

6. Ausência de melhor explicação neurológica.

III. E. M. clinicamente provável:

(sub-grupo: provável - primeiro surto)

1. História de sintomas recorrentes e remissivos, sugestivos de E. M., mas sem verificação objectiva de sintomatologia atribuível a lesão multifocal da substância branca do sistema nervoso central.

2. Verificação de um único surto, com sintomas resultantes de lesões múltiplas da substância branca do sistema nervoso central, boa recuperação, eventualmente seguidos de outra sintomatologia.

3. Alterações do L. C. R. sugestivas de E. M., mas sem obrigatoriedade de todos os aspectos mencionados no grupo anterior (E. M. com diagnóstico clinicamente definitivo).

4. Ausência de melhor explicação neurológica.

IV. E. M. clinicamente possivel:

(sub-grupo: possivel - primeiro surto)

1. História de sintomas recorrentes e remissivos, sem verificação.

2. Observação de sintomas insuficientes para evidenciarem mais do que uma lesão no sistema nervoso central.

3. As alterações do líquido céfalo-raquidiano características de E. M. não são obrigatórias.

4. Ausência de melhor explicação neurológica.

Os casos de nevrite retrobulbar monossintomática são incluídos neste grupo.

No esquema de Bauer, o relevo dado às alterações do L. C. R., nomeadamente aos aspectos imunitários, constitui um princípio de grande importância. Afigura-se, no entanto, excessivo considerar aquelas alterações obrigatórias para o diagnóstico clinicamente definitivo de E. M. Com efeito, no momento presente, não é possivel evidenciar uma reacção imunitária local, no sistema nervoso, na totalidade dos casos que apresentam probabilidade diagnóstica muito elevada, com base em exames do líquido céfalo-raquidiano, mesmo utilizando técnicas de alta sensibilidade na demonstração de bandas oligoclonais, como a electroforese em aga- 
rose e a focalização isoeléctrica (Hosein e Johnson, 1981). É certo que o uso de metodologia aperfeiçoada aproxima de $100 \%$ o número de casos com diagnóstico clínico considerado definitivo e alterações imunitárias características no líquido céfalo-raquidiano.

No esquema proposto por Bauer, nota-se a presença de um grupo de máxima probabilidade diagnóstica, representado pelos casos de E. M. comprovados por autópsia. Salienta-se a importância deste exame, quando efectuado no decurso das primeiras horas a seguir à morte, em muitos estudos biológicos. Também útil se mostra a inclusão dos sub-grupos $E$. $M$. provável - primeiro surto e $E$. M. possível - primeiro surto.

Com base nas ideias anteriormente expostas, temos vindo a utilizar o esquema de critérios de diagnóstico que a seguir se apresenta.

I. E. M. confirmada por autópsia.

II. E. M. com diagnóstico clínico definitivo:

1. Evolução recorrente e remissiva, com, pelo menos, dois surtos separados por intervalo não inferior a um mês; ou

2. Evolução progressiva, durante, pelo menos, seis meses.

3. Observação de sintomas neurológicos atribuíveis a lesão de mais de uma área do S. N. C., predominantemente da substância branca.

4. Frequentemente, alterações do L. C. R. características:

a) Pleocitose linfocitária, em geral, não superior a 50 células $/ \mathrm{mmc}$.

b) Indicação de síntese intratecal de imunoglobulinas.

5. Habitualmente, início das manifestações entre os dez e os cinquenta anos de idade.

6. Ausência de outro diagnóstico mais provável.

Neste grupo, individualiza-se o sub-grupo casos recentes: com duração aparente da doença não superior a um ano.

III. E. M. provável

1. História de sintomas recorrentes e remissivos, atribuíveis a lesão multifocal, predominantemente da substância branca do S. N. C., mas sem verificação objectiva; ou

2. Observação de sintomas de um único surto, atribuíveis, predominantemente, a lesões múltiplas da substância branca do S. N. C., com evolução remissiva.

3. Alterações do L. C. R. características de E. M., eventualmente presentes.

4. Ausência de diagnóstico mais provável.

De modo análogo ao presente no grupo anterior, considera-se a sub-divisão casos recentes, com duração aparente da doença não superior a um ano.
IV. E. M. possível

1. História de sintomas recorrentes e remissivos, sem verificação neurológica; ou

2. Observação de sintomas insuficientes para indicarem mais do que uma lesão, predominantemente, na substância branca do S. N. C.

3. Alterações do L. C. R. características de E. M., eventualmente presentes.

4. Ausência de diagnóstico mais provável.

Os casos de nevrite retrobulbar monossintomática são incluídos neste grupo. Também nele se individualizam os casos recentes, com duração aparente da doença não superior a um ano.

O esquema proposto segue a classificação de Bauer em quatro grupos de probabilidade diagnóstica. Atribui-se a devida importância às alterações do L. C. R., nomeadamente aos aspectos que indicam reacção imunitária intra-tecal e manifestam produção local, no sistema nervoso, de imunoglobulinas oligoclonais. Contudo, tanto estes aspectos como a pleocitose linfocitária, ainda que muito frequentemente presentes, não podem ser considerados obrigatórios.

Também, como no esquema de Bauer, se distinguem, nos diversos grupos, sub-grupos, entre nós designados casos recentes, nos quais se incluem, além de doentes considerados no primeiro surto, todos aqueles cujo início aparente da doença não ocorreu há mais de um ano.

Pensamos que, no futuro próximo, continuará a ser discutido o problema que nos ocupa, e que prosseguirá o aperfeiçoamento dos critérios de diagnóstico de E. M., certamente, em larga medida, com base no desenvolvimento de aquisições técnicas, dependentes da Neuro-imunologia e de outros domínios.

\section{BIBLIOGRAFIA}

1. SCHUMACHER G. A.; BEEBE, G.; KIBLER, R. F.; KURLAND, L. T.; KURTZKE, J. F.; MCDOWELL, F.; NAGLER, B.; SIBLEY, W. A.; TOURTELLOTTE, W. W.; WILLMON, T. L.: Problems of experimental trials of therapy in multiple sclerosis: Report by the panel on the evaluation of experimental trials of therapy in multiple sclerosis. Ann NY Acad. Sci. 1965; 122: 552 .

2. KURTZKE, J. F.: In International Symposium on Multiple Sclerosis. Acta Neurol. Scand. 1974: 50, Suppl. 58: 71.

3. ROSE, A. S.; ELLISON, G. W.; MYERS, L. W.; TOURTELLOTTE, W. W.: Criteria for the clinical diagnosis of multiple sclerosis. Neurology (Minneap) 1976; 26, n. ${ }^{\circ} 6$ - part 2: 20.

4. BAUER, H. J.: In Progress in MS - Research. An International Symposium. Göttingen, 1978. (Não publicado).

5. HOSEIN, Z. Z.; JOHNSON, K. P.: Isoelectric focusing of cerebrospinal fluid proteins in the diagnosis of multiple sclerosis. Neurology (Minneap) 1981; 31: 70.

Pedido de separatas: António Magalhães Serviço de Neurologia Hospital de Santa Maria 1600 Lisboa - Portugal 